Ophthalmologe 2022 $\cdot 119: 864-867$ https://doi.org/10.1007/s00347-021-01557-4

Eingegangen: 23. August 2021

Überarbeitet: 29. November 2021

Angenommen: 2. Dezember 2021

Online publiziert: 22. Dezember 2021

() Der/die Autor(en) 2021

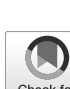

\section{2-jährige Patientin mit subakuter einseitiger Visusminderung}

\author{
M. Grünewald (D) · M. Maier \\ Klinik und Poliklinik für Augenheilkunde, Klinikum rechts der Isar der TU München, München, Deutschland
}

\section{Anamnese}

In unserer Ambulanz stellte sich eine 32jährige Patientin vor, die berichtete, seit 1 Woche am rechten Auge eine Visusminderung bemerkt zu haben. Zusätzlich seien am rechten Auge ein Schleier, Flimmern und schwarze Punkte zu sehen. Vorerkrankungen waren keine bekannt, auch wurde von der Patientin keine Dauermedikation eingenommen. Ein vorangegangener Infekt wurde ebenfalls verneint. In der Familie der Patientin waren keine Augenerkrankungen außer dem grauen Star bekannt. Bisherige Untersuchungen beim niedergelassenen Augenarzt waren immer regelrecht.

\section{Klinische Untersuchung}

Der bestkorrigierte Visus der myopen Patientin betrug rechts $0,7(-5,00)$, links 1,0 $\left(-5,00 /-0,50 / 26^{\circ}\right)$. Der Augeninnendruck war normoton. In der klinischen Untersuchung zeigte sich beidseits eine reizfreie Phakie mit klarer Linse. Im Glaskörper zeigten sich vereinzelte Zellen im Sinne einer milden Vitritis. Bei Betrachtung des Fundus fielen beidseits disseminierte gelbweißliche Herde vom hinteren Pol bis in die Peripherie auf (• Abb. 1). Die Papille präsentierte sich randscharf und vital, die Gefäße altersentsprechend und die Netzhaut allseits anliegend.

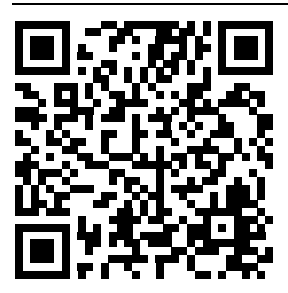

QR-Code scannen \& Beitrag online lesen
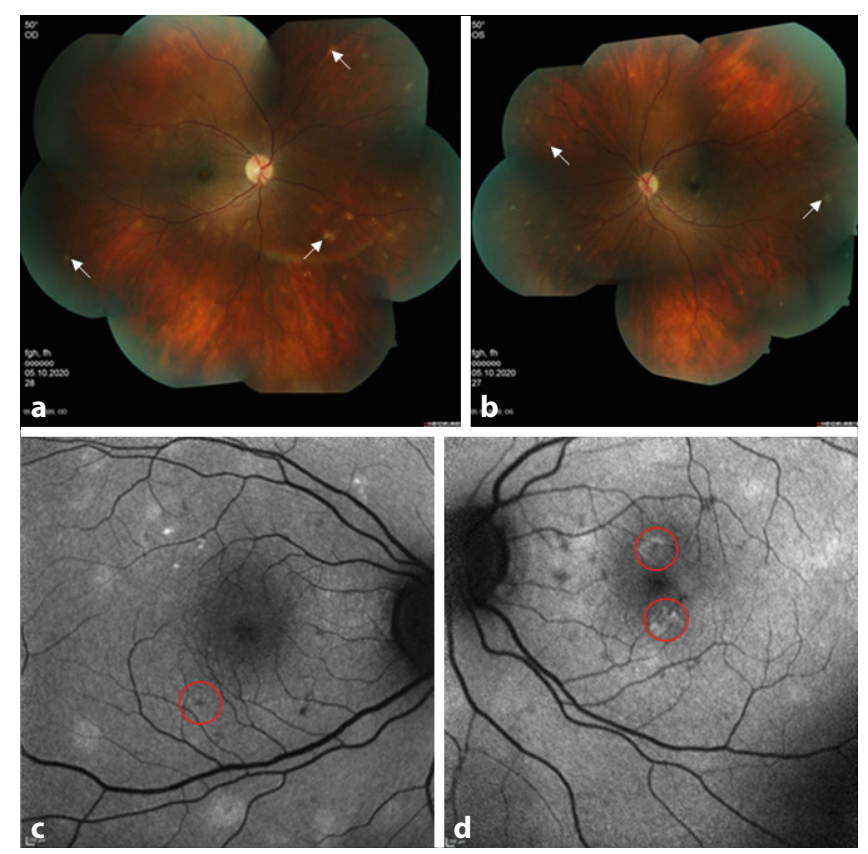

Abb. $1 \varangle$ Fundusfoto- und Fundusautofluoreszenzaufnahmen bei initialer Vorstellung. Rechtes $(\mathbf{a}, \mathbf{c})$ und linkes (b, d) Auge. a, c Vom hinteren Pol bis in die Peripherie gelbweißliche Läsionen (weiße Pfeile); in der Fundusautofluoreszenz $(\mathbf{c}, \mathbf{d})$ sind hypoautofluoreszente Läsionen mit teilweise hyperautofluoreszenten Ringen zu sehen (rote Kreise) 

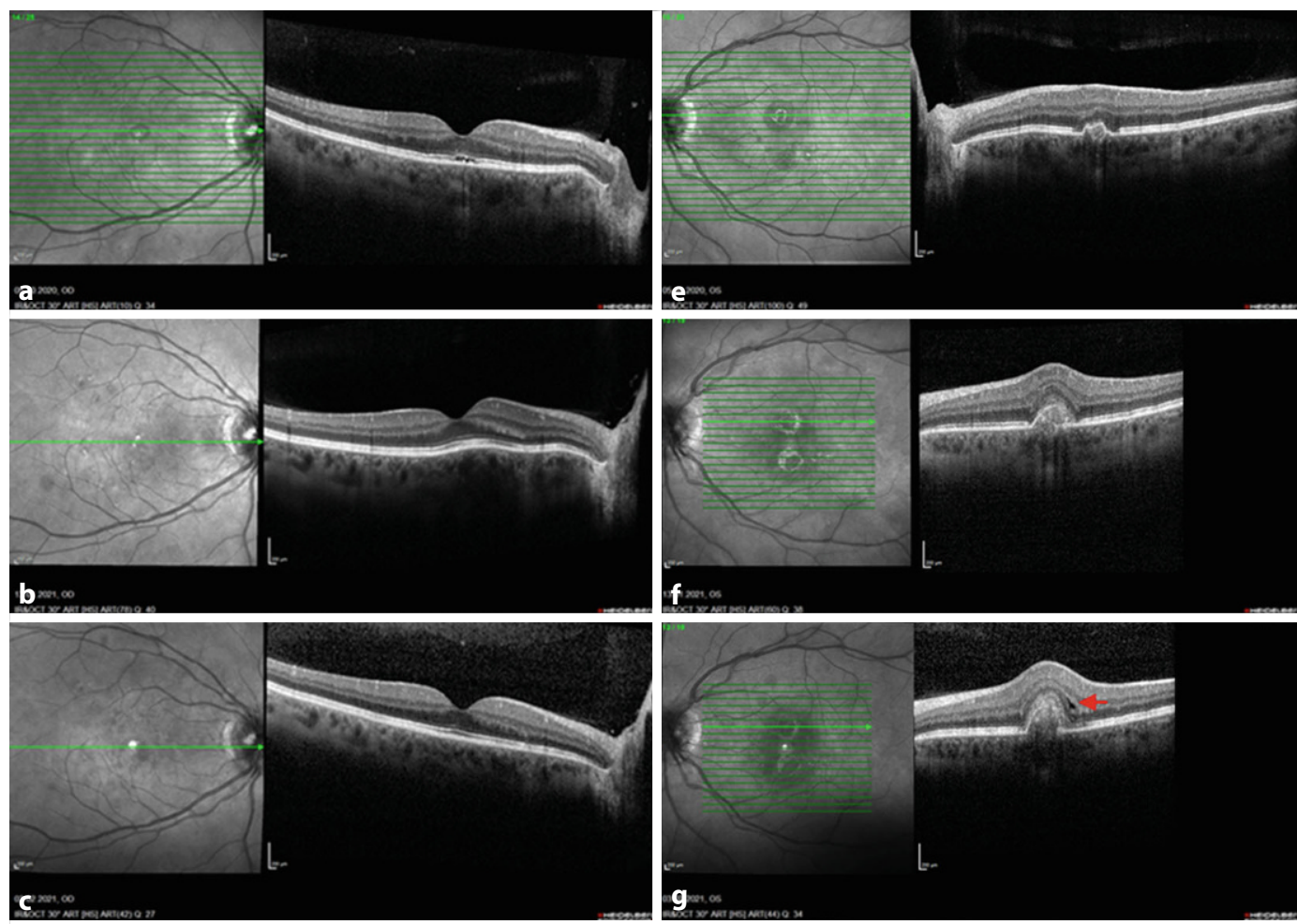

Abb. $2 \triangleleft$ SD-OCT-Aufnahmen im Verlauf. a-d rechtes Auge initial (a), nach 14 (b), 17 (c) und 28 (d) Wochen; e-h linkes Auge initial (e), nach 14 (f), 17 (g) und 28 (h) Wochen. a IS/OS-Destruktionen foveal; $\mathbf{b}$-d Regeneration der äußeren Netzhautschichten; $\mathbf{g}$ zentrale sekundäre CNV mit intraretinalen Flüssigkeitszysten roter Pfeil; $\mathbf{h}$ Rückgang der intraretinalen Flüssigkeit nach 3-maliger intravitrealer Injektion eines AntiVEGF(,vascular endothelial growth factor")-Medika-

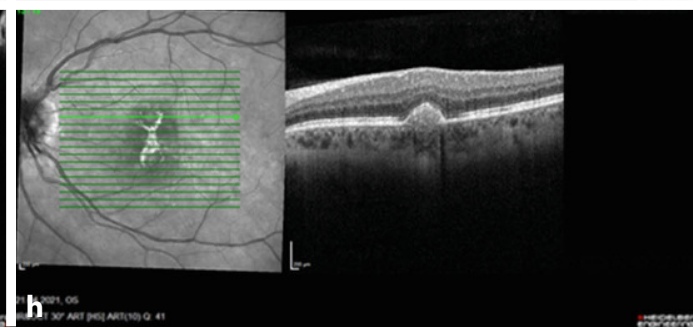
ments
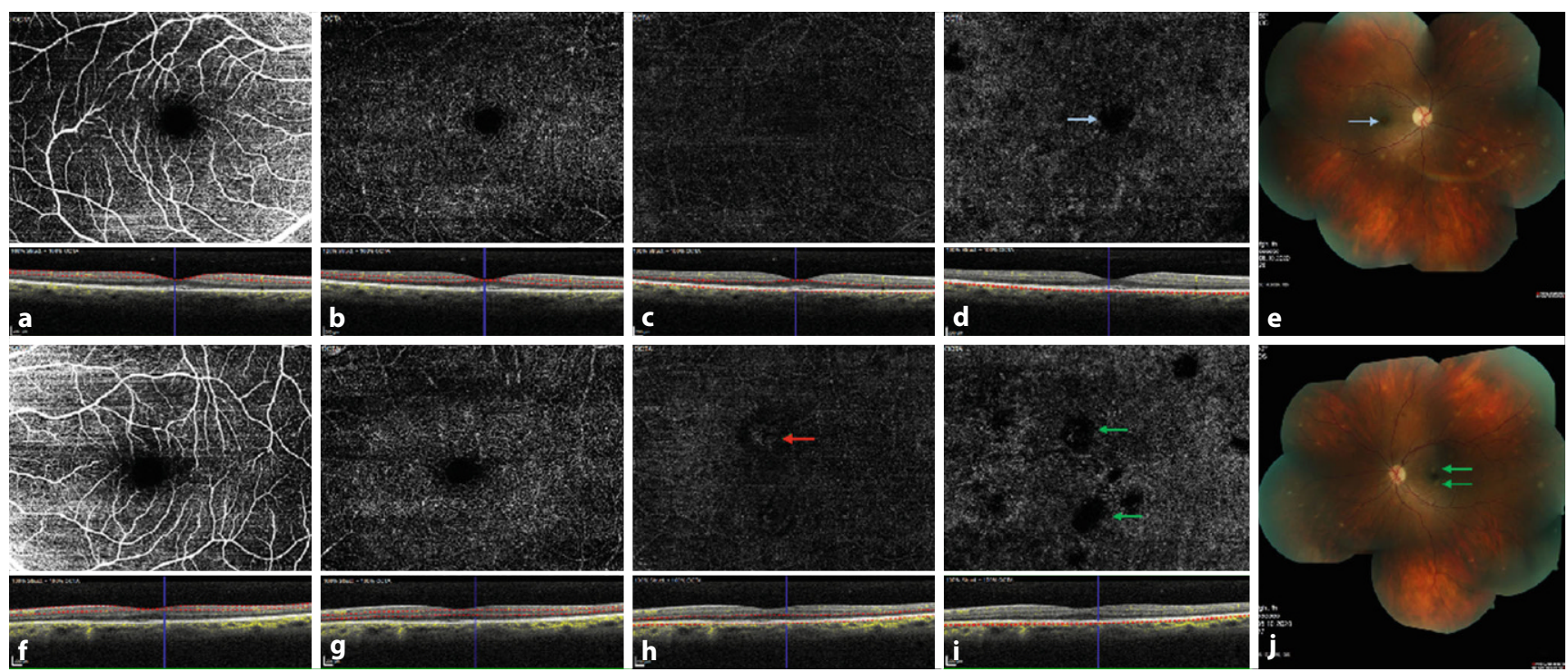

Abb. $3 \Delta$ OCT-Angiographie des rechten (a-e) und linken (f-j) Auges bei initialer Vorstellung. $\mathbf{a}$, $\mathbf{f O b e r f l a ̈ c h l i c h e r ~ r e t i n a l e r ~ G e - ~}$ fäßplexus, b, g tiefer retinaler Gefäßplexus, c, h avaskuläreZone, d, i Choriokapillaris, e, j Fundusfotografie. In h Gefäßmembran mit einem ringförmigen nicht perfundierenden Bereich (roterPfeil). In d und i Blockaden des Blutflusssignals in Choriokapillaris im Bereich der fundoskopisch auffälligen Herde $(\mathbf{e}, \mathbf{j})$ (hellblaue und grünePfeile) 

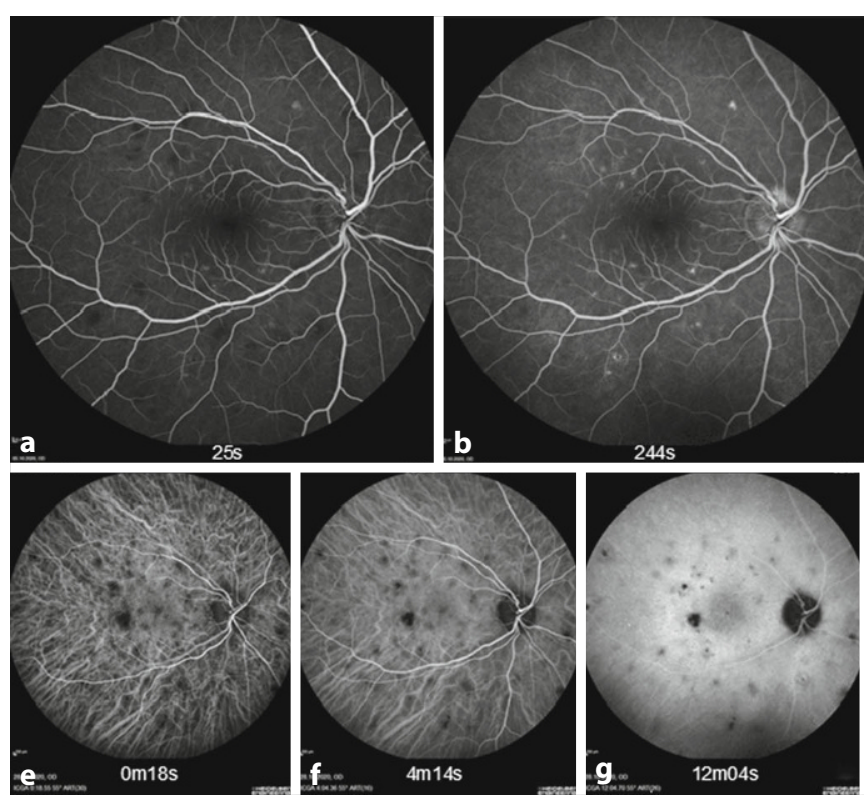
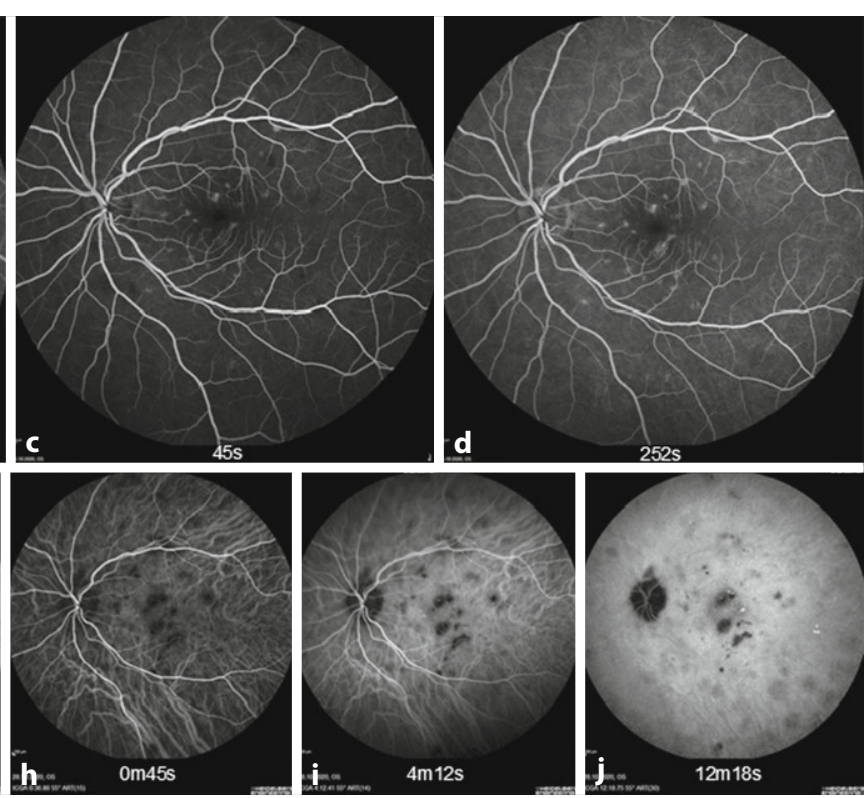

Abb. 4 A Fluoreszenz- (FLA) und Indocyaningrünangiographien (ICGA). In der FLA zeigen sich hypofluoreszente Areale in der Frühphase mit anschließenden Staining am rechten Auge (a, b), am linken Auge (c, d). In der ICGA präsentieren sich hypofluoreszente Herde in der Frühphase und in der intermediären Phase am rechten Auge (e, f), am linken Auge (h, i)

\section{Apparative Diagnostik}

Zur Diagnosefindung führten wir initial Fundusautofluoreszenz- und SD-OCT-Aufnahmen (Spectral-Domain-optische Kohärenztomographie) (Spectralis, Heidelberg Engineering), eine OCT-Angiographie und eine Fluoreszenzangiographie (FLA) durch.

Im SD-OCT fanden sich umschriebene Defekte der äußeren Netzhautschichten mit Destruktionen der inneren (IS) und äußeren (OS) Segmente der Fotore- zeptorschicht im Bereich der fundoskopisch auffälligen Herde, v. a. rechts foveal sowie linksseitig parafoveal (- Abb. 2a und e). In der Fundusautofluoreszenz zeigten sich hypoautofluoreszente Läsionen, teilweise mit hyperautofluoreszenten Ringen (- Abb. 1). In der OCT-Angiographie fanden sich Blockaden des Blutflusssignals in der Choriokapillaris, ebenfalls im Bereich der bereits beschriebenen auffälligen Herde. Die retinalen Schichten stellten sich regelrecht dar (• Abb. 3 und 4). In der FLA präsentierten sich die chorioretinalen Herde hypofluoreszent durch die Blockade des Fluoreszeins mit anschließendem Staining (- Abb. 4).

Zusätzlich erfolgten Blutuntersuchungen auf verschiedenste bakterielle, virale und parasitische Krankheitserreger, die alle unauffällig waren. Darüber hinaus erfolgte die Bestimmung des HLA-A29-Status der Patientin, welcher ebenfalls ein negatives Ergebnis erbrachte.

\section{Wie lautet Ihre Diagnose?}

\section{Diagnose}

In Zusammenschau der Ergebnisse der vielfältigen Untersuchungen ergab sich der Verdacht auf eine Erkrankung aus dem Formenkreis der White-Dot-Syndrome.

Zur weiteren Differenzierung führten wir eine Indocyaningrünangiographie (ICGA) durch. Bei dieser imponierten die beschriebenen chorioretinalen Herde hypofluoreszent (-Abb. 4). Leider musste die Untersuchung vorzeitig aufgrund von starkem Unwohlsein der Patientin abgebrochen werden, sodass die Spätphase nicht abschließend beurteilbar war.
Der Begriff „White-Dot-Syndrom", erstmals geprägt von Jampol et al. im Jahr 1984, beschreibt eine heterogene Gruppe von Erkrankungen, die sich durch die namengebenden weißen Flecken in der Fundoskopie auszeichnen. Die Ätiologie der Erkrankungen ist nicht endgültig geklärt, diskutiert werden sowohl autoimmune als auch infektiöse Auslöser. Eine Differenzierung der verschiedenen Krankheitsentitäten ist nicht immer komplett möglich, so können sich z. B. punktförmige innere Choroidopathie (PIC) und multifokale Chorioiditis (MFC) ähnlich präsentieren [4].
Im Falle der hier vorgestellten Patientin ist die wahrscheinlichste Diagnose eine MFC aufgrund der milden Vitritis, des beidseitigen Auftretens und der typischen Verteilung der chorioretinalen Herde am gesamten Fundus. Vor allem die Verteilung der chorioretinalen Herde vom hinteren Pol bis in die Peripherie ermöglicht eine Differenzierung zur PIC. Im Gegensatz zur Birdshot-Retinochoroidopathie (BSRC) zeigten sich die beschriebenen eher kleinen 50-350 $\mu$ m durchmessenden Läsionen (bei BSCR typischerweise 500-1500 $\mu \mathrm{m}$ ) und in der FLA keine retinalen Gefäßleckagen im Sinne einer retinalen Vaskulitis. Auch war der HLA-A29-Status, 
der in über $95 \%$ der Fälle einer BSCR positiv ist, negativ.

Zusätzlich passen die typischen Befunde in FLA (Herde in Frühphase hypofluoreszent mit zunehmendem Staining bis in die Spätphase) und ICGA (hypofluoreszente Herde) zur Verdachtsdiagnose MFC [3, 4].

\section{॥ Diagnose: Multifokale Chorioiditis}

Die MFC betrifft überwiegend myope Frauen mittleren Alters, der Altersgipfel liegt bei ca. 40 bis 50 Jahren. Sie verläuft meist bilateral, häufig aber asymmetrisch und schubweise, sodass initial der Eindruck entstehen kann, dass nur 1 Auge betroffen ist $[2,4]$. Zu den Differenzialdiagnosen gehören einerseits andere White-Dot-Syndrome, allen voran die PIC und die BSRC. Andererseits können auch infektiöse Ursachen eine ähnliche Symptomatik hervorrufen, z. B. die okuläre Histoplasmose [1].

Die MFC ist charakterisiert durch eine milde Vitritis, die oben schon beschriebenen gelbweißen Läsionen im RPE-Niveau und drusenartiges Material zwischen RPE und Bruch-Membran mit IS-/OS-Destruktionen [5].

Nach Abheilung eines akuten Schubes können sich ausgeprägte atrophische Narben bilden. Als Spätkomplikation kann es in 32-46\% der Erkrankungen zur Bildung von sekundären inflammatorischen choroidalen Neovaskularisationen (CNVs) kommen, infolge derer sich subretinale Fibrosen bilden können [4].

\section{Therapie}

Zur Therapie begannen wir mit einer körpergewichtsadaptierten Kortisontherapie (1 mg/kgKG), welche über einen Zeitraum von 4 Wochen von $60 \mathrm{mg}$ auf eine Erhaltungsdosis von $5 \mathrm{mg}$ reduziert wurde. Unter der Kortisontherapie zeigte sich nach 4 Wochen eine deutliche Befundbesserung mit Steigerung des bestkorrigierten Visus am rechten Auge auf 0,9. Die äußeren Netzhautschichten am rechten Auge waren wieder regelrecht konfiguriert (- Abb. 2).

Im weiteren Verlauf zeigte sich der Visus am rechten Auge stabil bei $0,9-1,0$ ohne zusätzliche Symptome. Drei Monate nach Erstvorstellung und unter der Kortisonerhaltungsdosis von $5 \mathrm{mg}$ bemerkte die Patientin die Symptome, wegen derer sie sich initial vorgestellt hatte, auch am linken Auge. Daraufhin erfolgte die erneute Erhöhung der Kortisontherapie. Bei schlechter Verträglichkeit der Kortisondauertherapie wurde die Therapie in Zusammenarbeit mit den Kollegen der Rheumatologie auf Mycophenolat-Mofetil 500 mg 2-mal täglich umgestellt.

Im weiteren Verlauf entwickelte sich am linken Auge eine zentrale choroidale Neovaskularisation mit Visusabfall auf 0,4 , die mittels mehrfacher VEGF-Injektion behandelt wurde. Nach 3-maliger intravitrealer Injektion zeigte sich eine Verbesserung des Visus auf zuletzt 0,8 mit Rückgang der intraretinalen Flüssigkeit (• Abb. 2h). Es erfolgen weiterhin regelmäßige Kontrollen inklusive SD-OCT-Aufnahmen, um erneute Krankheitsschübe frühzeitig zu erkennen und zu therapieren.

\section{Fazit für die Praxis}

Seltene White-Dot-Syndrome wie die multifokale Chorioiditis benötigen eine differenzierte multimodale Diagnostik sowie engmaschige Kontrollen. Eine Therapie mit Kortison und anderen immunmodulatorischen Substanzen ist das Mittel der Wahl, um eine Remission zu erzielen. Eine gefürchtete Komplikation ist die Entwicklung von sekundären inflammatorischen choroidalen Neovaskularisationen, die jedoch gut auf VEGFInhibitoren ansprechen.

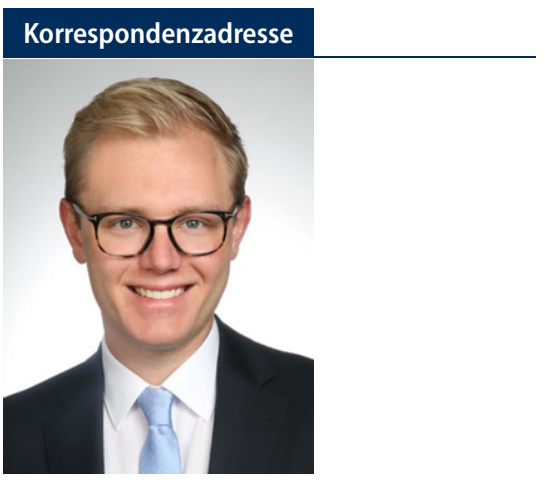

Dr. med. sci. M. Grünewald

Klinik und Poliklinik für Augenheilkunde, Klinikum rechts der Isar der TU München Ismaninger Str. 22, 81675 München, Deutschland marc.gruenewald@mri.tum.de
Funding. Open Access funding enabled and organized by Projekt DEAL.

\section{Einhaltung ethischer Richtlinien}

Interessenkonflikt. M. Maier: Vergütung auf Honorarbasis bei Allergan, Bayer, Novartis, Heidelberg Engineering. M. Grünewald gibt an, dass kein Interessenkonflikt besteht.

Für diesen Beitrag wurden von den Autoren keine Studien an Menschen oder Tieren durchgeführt. Für die aufgeführten Studien gelten die jeweils dort angegebenen ethischen Richtlinien. Für Bildmaterial ode anderweitige Angaben innerhalb des Manuskripts, über die Patienten zu identifizieren sind, liegt von ihnen und/oder ihren gesetzlichen Vertretern eine schriftliche Einwilligung vor.

Open Access. Dieser Artikel wird unter der Creative Commons Namensnennung 4.0 International Lizenz veröffentlicht, welche die Nutzung, Vervielfältigung, Bearbeitung, Verbreitung und Wiedergabe in jeglichem Medium und Format erlaubt, sofern Sie den/die ursprünglichen Autor(en) und die Quelle ordnungsgemäß nennen, einen Link zur Creative Commons Lizenz beifügen und angeben, ob Änderungen vorgenommen wurden.

Die in diesem Artikel enthaltenen Bilder und sonstiges Drittmaterial unterliegen ebenfalls der genannten Creative Commons Lizenz, sofern sich aus der Abbildungslegende nichts anderes ergibt. Sofern das betreffende Material nicht unter der genannten Creative Commons Lizenz steht und die betreffende Handlung nicht nach gesetzlichen Vorschriften erlaubt ist, ist für die oben aufgeführten Weiterverwendungen des Materials die Einwilligung des jeweiligen Rechteinhabers einzuholen.

Weitere Details zur Lizenz entnehmen Sie bitte der Lizenzinformation auf http://creativecommons.org/ licenses/by/4.0/deed.de.

\section{Literatur}

1. Dreyer RF, Gass DJ (1984) Multifocal choroiditis and panuveitis. A syndrome that mimics ocular histoplasmosis. Arch Ophthalmol 102(12):1776-1784

2. Fung AT, Pal S, Yannuzzi NA et al (2014) Multifocal choroiditis without panuveitis: clinical characteristics and progression. Retina 34(1):98-107

3. Pohlmann D, Winterhalter S, Pleyer U (2019) White-dot-Syndrome: Grundlagen, Diagnostik und Therapie. Ophthalmologe. https://doi.org/10 1007/s00347-019-01012-5

4. Quillen DA, Davis JB, Gottlieb JL, Blodi BA, Callanan DG, Chang TS, Equi RA (2004) The white dot syndromes. Am J Ophthalmol 137(3):538-550

5. Vance SK, Khan S, Klancnik JM, Freund KB (2011) Characteristic spectral-domain optical coherence tomography findings of multifocal choroiditis. Retina 31(4):717-723 